\title{
Genetic relationships between ecologically divergent species of talitrid amphipod (Crustacea)
}

\author{
M. B. Conceição*, J. D. D. Bishop**, J. P. Thorpe*** \\ The University of Liverpool, Port Erin Marine Laboratory, Port Erin, Isle of Man, IM9 6JA, United Kingdom
}

\begin{abstract}
Allozyme electrophoresis was used to investigate the genetic relationships between several species of the amphipod family Talitridae representative of different ecomorphological groups. The analysis of 21 putative gene loci revealed that in general the species studied have relatively low levels of genetic variability when compared with published data for other crustaceans, including other amphipod species. The genetic identities between the species studied were in general accord with existing taxonomy and in good agreement with the broad relationship expected between taxonomic divergence and genetic identity. Dendrograms produced by UPGMA and Wagner clustering procedures differed in the position of 1 species. However, both dendrograms suggested that species of the genera Orchestia (beachfleas) and Talorchestia (sandhoppers) are more closely related to each other than to Talitrus saltator (also a sandhopper), and hence the results did not show a clear genetic divergence between the different ecological groups of talitrids. The analyses supported the taxonomic decision of previous authors to remove the genus Hyale from the family Talitridae. However, the levels of genetic divergence found within the family are too low to reconcile readily with the proposed radiation of the group during the Cretaceous period and indicate that the talitrids probably evolved considerably more recently.
\end{abstract}

KEY WORDS: Talitrids - Genetics · Allozymes - Amphipods

\section{INTRODUCTION}

Talitrid amphipods, along with the larvae of seaweed flies, are the dominant invertebrates of macrophytic detritus (wrack) cast up on the strand line of both soft and hard shores. These groups accordingly act as the most important macrofaunal consumers of cast algae on temperate shores of both the northern and southern hemispheres (e.g. Griffiths \& StentonDozey 1981, Moore \& Francis 1985). Talitrids may be the predominant consumers of algal floating wrack (aevja) (Robertson \& Lucas 1983), of Spartina spp. litter in saltmarshes (Levinton et al. 1977) and of turtle grass debris in the tropics (Venables 1981). Detritus from the breakdown of the strand line may constitute an impor-

\footnotetext{
- Present address: Lab. Bioquímica Marinha, Depto. Química, Fundação Universidade do Rio Grande, CP 474, Rio Grande, RS-96201-900, Brazil

- Present address: Marine Biological Association of the UK,

The Laboratory, Citadel Hill, Plymouth PL1 2PB, UK

...E-mail: peml@liverpool.ac.uk
}

tant source of food intertidally, for instance for the fauna of sandy beaches, while derived nutrients may contribute to primary production in nearshore areas (e.g. Griffiths \& Stenton-Dozey 1981, Koop et al. 1982a, b). Talitrids are early colonisers of strand debris and may reach extremely high population densities (e.g. Griffiths \& Stenton-Dozey 1981) and levels of production (Venables 1981). They are exploited by a large range of predators, including fish, birds, mammals and other wrack invertebrates (reviewed by Wildish 1988). The family Talitridae exhibits many adaptations for semi-terrestrial and terrestrial life, and is unique among amphipods in having fully terrestrial representatives (Hurley 1959). In those parts of the world where they occur, euterrestrial talitrids (landhoppers) may play a dominant role in the exploitation of land-plant debris in forest-floor leaf litter communities which parallels that of their maritime counterparts (Bousfield 1984).

The Talitridae, with more than 200 described species, constitute the largest family within the superfam- 
ily Talitroidea (Bousfield 1982). Despite advances in taxonomy and many additional distributional records in recent years, major problems remain with the systematics of the group. From distributional data it has been concluded that the Indo-Pacific region of the southern hemisphere is the epicentre of talitroid species diversity (Bousfield 1984). Bousfield proposed that talitrids evolved during the Cretaceous Period and early Cenozoic Era from aquatic ancestors, allied to primitive genera of the family Hyalidae; the break-up of Pangaea into many continents, providing new coastal regions, and the later evolution of angiosperm forests, providing food resources, stimulated the geographical and evolutionary radiation of the talitrids. Bousfield (1984) also subdivided the family Talitridae into 4 loose systematic-ecological groups: palustral species, which he considered morphologically primitive and which occur in marine, estuarine, and some freshwaler habitats; beachfleas, which are more advanced species, occur in supralittoral habitats and coastal rain forests, and are non-substrate-modifying; sandhoppers, which are supralittoral substrate-modifying (burrowing) species found on sandy beaches; and landhoppers, which are specialised terrestrial species occupying forest leaf litter.

These commonly used ecomorphological sub-groups of the family are informal and probably polyphyletic (Bousfield 1982, 1984). For instance, within the landhoppers Bousfield recognised 2 assemblages, simplidactylate and cuspidactylate forms, with separate evolutionary origins. According to Bousfield (1984) the simplidactylate landhoppers evolved from palustral ancestors in the southern hemisphere, and the cuspidactylate landhoppers are thought to be derived from beachflea ancestors of tropical and warm-temperate coastal regions.

Recently some attention has been given to phylogenetic relationships within talitrids, although none of the work has used any molecular techniques. From a cladistic analysis of gammaridean morphology Kim \& Kim (1993) concluded that the Talitroidea were monophyletic. Lindeman (1991) used phenetic and cladistic analysis of morphological data in a phylogenetic study of neotropical landhoppers and Moore et al. (1993) used the morphology of the antennary gland exit duct in an ecological series of talitroids to assess whether the differences in form support Bousfield's hypothesis.

The study of enzyme variation has long been used as a tool in population genetics and also to provide taxonomic information on a wide range of organisms including many invertebrates (reviewed by, e.g., Thorpe \& Solé-Cava 1994; see Stewart 1993 for review of amphipod data). The few such studies of talitrids (McDonald 1985, 1987, 1991, Bulnheim \& Scholl 1986, De Mathaeis et al. 1994) were not concerned with phy-

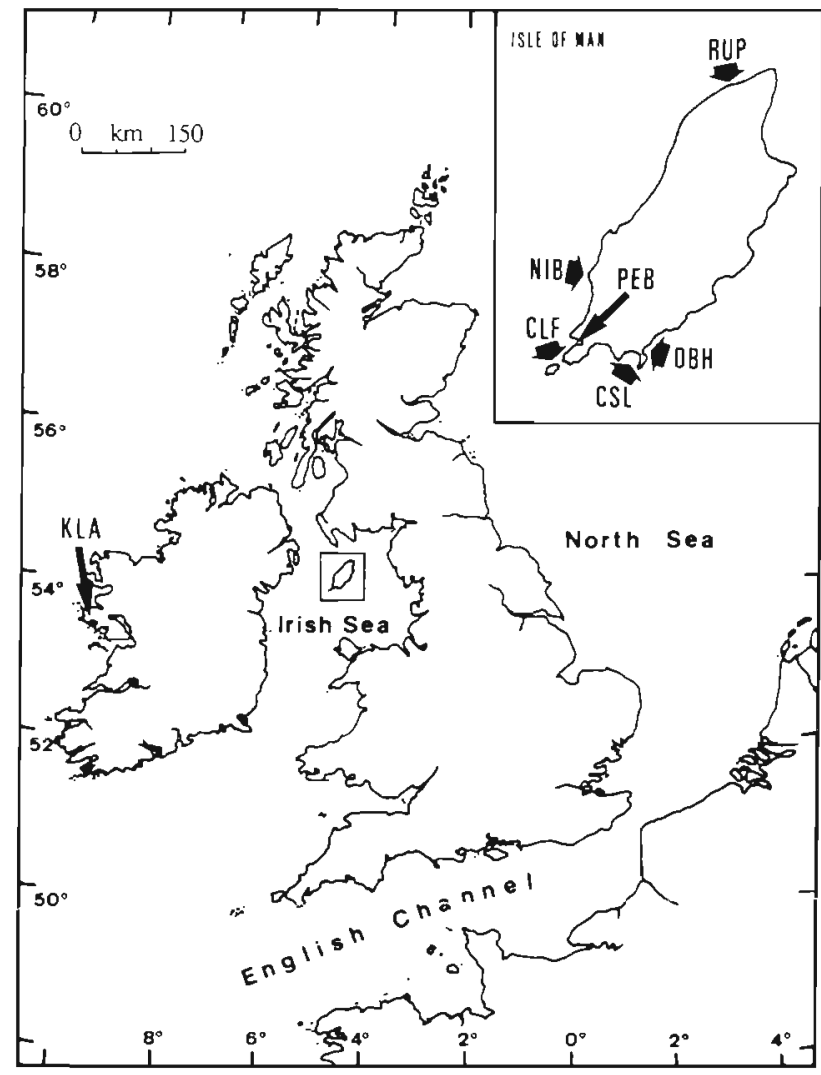

Fig. 1. British Isles: inset shows Isle of Man sampling sites. Site names are given in full in Table 1

logeny. The present study investigates genetic relationships between some of the talitrid ecological groups defined by Bousfield (1984). To put relationships within the family in context, additional comparisons are made within the superfamily Talitroidea and with one non-talitroid amphipod species.

\section{MATERIALS AND METHODS}

Sampling and collecting sites. The landhopper Arcitalitrus dornieni was collected amongst leaf litter. All other talitroids and Gammarus crinicornis were taken from the intertidal and supralittoral regions of sandy or rocky shores, and were collected among algae or sand. Samples were maintained alive in the laboratory until required for electrophoresis. The species studied and the sampling sites (Fig. 1) are listed in Table 1.

The choice of the 2 non-talitrid species for comparisons outside the Talitridae followed from previous work. Thus, an intertidal species of Hyale was used for comparison as it is regarded as ecologically analogous and phylogenetically close to the probable ancestor of the Talitridae (Bousfield 1984). The genus Hyale has 
Table 1. Talitrids and other amphipods used in this study, and the ecological type (for talitrids only; after Bousfield 1984) and sampling locality (see Fig. 1) for each species

\begin{tabular}{|c|c|c|c|c|}
\hline Species & Ecological type & Family & Sampling site & Location \\
\hline $\begin{array}{l}\text { Talitrus saltator } \\
\text { (Montagu, 1808) }\end{array}$ & Sandhopper & Talitridae & Derbyhaven (DBH) & Isle of Man \\
\hline $\begin{array}{l}\text { Talorchestia deshayesii } \\
\text { (Audouin, 1826) }\end{array}$ & Sandhopper & Talitridae & Castletown Bay, Langness (CSL) & Isle of Man \\
\hline $\begin{array}{l}\text { Orchestia gammarellus } \\
\text { (Pallas, } 1766 \text { ) }\end{array}$ & Beachflea & Talitridae & Niarbyl (NIB) & Isle of Man \\
\hline $\begin{array}{l}\text { Orchestia mediterranea } \\
\text { Costa, } 1857\end{array}$ & Beachflea & Talitridae & Calf of Man (CLF) & Isle of Man \\
\hline $\begin{array}{l}\text { Arcitalitrus dorrieni } \\
\text { (Hunt, 1925) }\end{array}$ & Simplidactylate landhopper & Talitridae & Kylemore Abbey (KLA) & $\begin{array}{l}\text { Co. Galway, } \\
\text { Ireland }\end{array}$ \\
\hline $\begin{array}{l}\text { Hyale nilssoni } \\
\text { (Rathke, } 1843 \text { ) }\end{array}$ & - & Hyalidae & Port Erin Bay (PEB) & Isle of Man \\
\hline $\begin{array}{l}\text { Gammarus crinicornis } \\
\text { Stock, } 1966\end{array}$ & - & Gammaridae & Rue Point (RUP) & Isle of Man \\
\hline
\end{tabular}

been used to define the plesiomorphic state for the phylogenetic analyses of talitrid morphological characters by Bousfield (1984), Lindeman (1991), and Moore et al. (1993). It was also taken as the starting point in the review of physiological adaptations of talitrids to semi-terrestrial and terrestrial environments by Spicer et al. (1987). The non-talitroid family Gammaridae has been considered to include a pool of species representing the most primitive kind of gammaridean amphipod (Barnard 1974), although this view is by no means universally held (e.g. Bousfield 1984). The well-studied genus Gammarus has been the subject of many electrophoretic investigations (see Stewart 1993), and a species of the genus was considered to represent the

Table 2. The 13 enzyme systems scored in all species in this study, with E.C. numbers, buffers used, and number of loci studied. See 'Materials and methods, Electrophoretic analysis' for numbering of buffer systems

\begin{tabular}{|lccc|}
\hline Enzyme (abbreviation) & $\begin{array}{c}\text { E.C. } \\
\text { number }\end{array}$ & $\begin{array}{c}\text { Buffer } \\
\text { system }\end{array}$ & $\begin{array}{c}\text { No. of } \\
\text { loci }\end{array}$ \\
\hline Acid phosphatase (ACP) & 3.1 .3 .2 & 2 & 1 \\
Aspartate aminotransferase (AAT) & 2.6 .1 .1 & 2 & 2 \\
Diaphorase (DIA) & 1.8 .1 .4 & 2 & 3 \\
Fumarase (FUM) & 4.2 .1 .2 & 1 & 1 \\
Glucose-6-phosphate & 5.3 .1 .9 & 1 & 1 \\
isomerase (GPI) & & & \\
Hexokinase (HK) & 2.7 .1 .1 & 1 & 1 \\
Lactate dehydrogenase (LDH) & 1.1 .1 .27 & 1 & 1 \\
Leucine aminopeptidase (LAP) & 3.4 .11 .1 & 3 & 2 \\
Malate dehydrogenase (MDH) & 1.1 .1 .37 & 1 & 3 \\
Malic enzyme (ME) & 1.1 .1 .40 & 3 & 2 \\
Mannose-6-phosphate & 5.3 .1 .8 & 1 & 1 \\
isomerase (MPI) & & & \\
Peptidase (PEP) & 3.4 .13 .11 & 3 & 2 \\
Phosphoglucomutase (PGM) & 5.4 .2 .2 & 1 & 1 \\
\hline
\end{tabular}

typical condition of aquatic gammarideans in the comparative study of talitrids by Moore et al. (1993).

Electrophoretic analysis. Electrophoresis followed standard methods (Harris \& Hopkinson 1976, Murphy et al. 1990). The homogenisation buffer used was $0.2 \mathrm{M}$ Tris- $\mathrm{HCl} \mathrm{pH}$ 8.0. The homogenates were centrifuged at $3000 \times g$ for $3 \mathrm{~min}$. Full details are given in Conceiçāo (1995).

The buffer systems utilised were (1) Tris-citrate, pH 8.0 (Siciliano \& Shaw 1976), (2) discontinuous Trisborate-citrate, pH 8.2-8.7 (Poulik 1957), and (3) lithium hydroxide, pH 8.15-8.30 (Shaklee \& Keenam 1986). Thirty-one enzyme systems were tested, of which 13 could be scored in all species (see Table 2). Talitrus saltator was used as a standard for the measurement of the relative mobilities of alleles for all species.

The program BIOSYS-1 (Swofford \& Selander 1981) was used to estimate the variability within species and genetic differentiation among species. Nei's (1978) unbiased genetic distance was used to construct a UPGMA phenogram (Sneath \& Sokal 1973), and the Prevosti distance (Wright 1978) was employed to estimate the phylogenetic relationship through the Wagner procedure (Farris 1972).

Goodness of fit statistics were chosen as the primary criteria for evaluating which tree-building method produced the better tree. The statistics employed were: the $f$ of Farris (1972), the $F$ of Prager \& Wilson (1976), the percent standard deviation $(\% S D)$ of Fitch \& Margoliash (1967), and the cophenetic correlation (CC) of Sneath \& Sokal (1973). These statistics are intended to estimate the degree to which the output (tree) distances between taxa reflect the corresponding input distances, indicating the amount of homoplasy between a pair of taxa (Farris 1972), and are often used to choose between alternative trees generated by different methods (Swofford 1981). 
Table 3. Allele frequencies in the 7 amphipod species used in this study. (1) Talitrus saltator, (2) Talorchestia deshayesij. (3) Orchestia gammarellus, (4) Orchestia mediterranea, (5) Arcitalitrus dorrieni, (6) Hyale nilssoni, (7) Gammarus crinicorris. N shows the number of specimens used

\begin{tabular}{|c|c|c|c|c|c|c|c|c|c|c|c|c|c|c|c|}
\hline $\begin{array}{l}\text { Locus, } \\
\text { Alleles }\end{array}$ & \multicolumn{7}{|c|}{ Species } & \multicolumn{4}{|l|}{ Locus, } & $\begin{array}{c}\text { Species } \\
4\end{array}$ & Species & 6 & 7 \\
\hline$G P I^{\circ}$ & & & & & & & & $M D H$ & - & led) & & & & & \\
\hline$(\mathrm{N})$ & (59) & $(70)$ & $(48)$ & (33) & $(62)$ & $(45)$ & (39) & 100 & 1.000 & 0.000 & 0.000 & 1.000 & 0.000 & 0.000 & 0.000 \\
\hline 39 & 0.000 & 0.000 & 0.000 & 0.000 & 0.000 & 0.024 & 0.000 & 180 & 0.000 & 0.000 & 0.000 & 0.000 & 0.000 & 0.000 & 1.000 \\
\hline 56 & 0.000 & 0.000 & 0.000 & 0.000 & 0.000 & 0.976 & 0.000 & $M D H$ & & & & & & & \\
\hline 58 & 0.000 & 0.000 & 0.010 & 0.045 & 0.000 & 0.000 & 0.000 & $(\mathrm{~N})$ & $(30)$ & (30) & (30) & (33) & (45) & (45) & (21) \\
\hline 67 & 0.000 & 0.336 & 0.990 & 0.955 & 0.000 & 0.000 & 0.000 & 85 & 0.000 & 0.000 & 0.000 & 0.000 & 0.000 & 1.000 & 0.000 \\
\hline 80 & 0.000 & 0.000 & 0.000 & 0.000 & 0.000 & 0.000 & 0.064 & 90 & 0.000 & 1.000 & 0.000 & 0.000 & 1.000 & 0.000 & .000 \\
\hline 85 & 0.008 & 0.664 & 0.000 & 0.000 & 0.000 & 0.000 & 0.000 & 95 & 0.000 & 0.000 & 1.000 & 0.000 & 0.000 & 0.000 & .000 \\
\hline 100 & 0.898 & 0.000 & 0.000 & 0.000 & 0.000 & 0.000 & 0.910 & 10 . & 1.000 & 0.000 & 0.000 & 1.000 & 0.000 & 0.000 & .000 \\
\hline 113 & 0.000 & 0.000 & 0.000 & 0.000 & 1.000 & 0.000 & 0.026 & & 0.000 & 0.000 & 0.000 & 0.000 & 0.000 & .000 & .000 \\
\hline 115 & 0.093 & 0.000 & 0.000 & 0.000 & 0.000 & 0.000 & 0.000 & & & & & & & & \\
\hline $\begin{array}{l}P G M^{\circ} \\
\text { (N) }\end{array}$ & (49) & (62) & $(48)$ & (31) & $(65)$ & $(48)$ & (45) & $(\mathrm{N})$ & $(30)$ & 100 & 1.0. & & (Fiv) & & \\
\hline 65 & 0.000 & 0.016 & 0.000 & 0.000 & 0.000 & 0.000 & 0.000 & 70 & 0.000 & 0.000 & 0.000 & 0.000 & 0.000 & 1.000 & 0.000 \\
\hline 80 & 0.000 & 0.435 & 0.000 & 0.000 & 0.000 & 0.000 & 0.000 & 80 & 0.000 & 0.000 & 1.000 & 0.000 & 0.000 & 0.000 & 0.000 \\
\hline 87 & 0.020 & 0.540 & 0.000 & 0.000 & 0.000 & 0.000 & 0.000 & 90 & 0.000 & 1.000 & 0.000 & 0.000 & 1.000 & 0.000 & 0.000 \\
\hline 90 & 0.112 & 0.008 & 0.000 & 0.032 & 0.000 & 0.000 & 0.000 & 95 & 0.000 & 0.000 & 0.000 & 0.000 & 0.000 & 0.000 & 1.000 \\
\hline 95 & 0.000 & 0.000 & 0.000 & 0.000 & 0.000 & 1.000 & 0.000 & 98 & 0.000 & 0.000 & 0.000 & 1.000 & 0.000 & 0.000 & 0.000 \\
\hline 100 & 0.837 & 0.000 & 0.990 & 0.839 & 0.979 & 0.000 & 0.000 & 100 & 1.000 & 0.000 & 0.000 & 0.000 & 0.000 & 0.000 & 0.000 \\
\hline 102 & 0.000 & 0.000 & 0.000 & 0.000 & 0.000 & 0.000 & 0.100 & $M E-1^{\circ}$ & & & & & & & \\
\hline 105 & 0.000 & 0.000 & 0.000 & 0.000 & 0.021 & 0.000 & 0.000 & $(\mathrm{~N}$ & (30) & (30) & $(30)$ & (33) & $(42)$ & $(48)$ & (21) \\
\hline 108 & 0.000 & 0.000 & 0.000 & 0.000 & 0.000 & 0.000 & 0.900 & 75 & 0.000 & 0.000 & 0.000 & 0.000 & 1.000 & 0.000 & 0.000 \\
\hline 110 & 0.031 & 0.000 & 0.010 & 0.129 & 0.000 & 0.000 & 0.000 & 98 & 0.000 & 0.000 & 0.000 & 1.000 & 0.000 & 0.000 & 0.000 \\
\hline$M P I^{\circ}$ & & & & & & & & 100 & 1.000 & 1.000 & 0.000 & 0.000 & 0.000 & 0.000 & 0.000 \\
\hline$(\mathrm{N})$ & (30) & (42) & $(47)$ & (30) & $(62)$ & (45) & (39) & 102 & 0.000 & 0.000 & 1.000 & 0.000 & 0.000 & 0.000 & 0.000 \\
\hline 85 & 0.000 & 0.000 & 0.160 & 0.050 & 0.000 & 0.113 & 0.000 & 120 & 0.000 & 0.000 & 0.000 & 0.000 & 0.000 & 1.000 & 1.000 \\
\hline 90 & 0.000 & 0.000 & 0.000 & 0.000 & 1.000 & 0.000 & 0.000 & $\Lambda$ & & & & & & & \\
\hline 95 & 0.000 & 0.988 & 0.830 & 0.950 & 0.000 & 0.000 & 0.000 & & $(30)$ & & $(3$ & (3 & 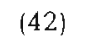 & 3) & (21) \\
\hline 98 & 0.000 & 0.000 & 0.000 & 0.000 & 0.000 & 0.000 & 0.038 & & 1.000 & 1.000 & 1.000 & 1.000 & 1.000 & 1.000 & 1.000 \\
\hline 100 & 1.000 & 0.012 & 0.011 & 0.000 & 0.000 & 0.887 & 0.000 & & & & & & & & \\
\hline 102 & 0.000 & 0.000 & 0.000 & 0.000 & 0.000 & 0.000 & 0.115 & $\begin{array}{l}\text { LDF } \\
(\mathrm{N})\end{array}$ & $(30)$ & (30) & $(30)$ & $(3)$ & $(40)$ & $(48)$ & (21) \\
\hline 105 & 0.000 & 0.000 & 0.000 & 0.000 & 0.000 & 0.000 & 0.692 & 90 & 0.000 & 0.000 & 1.000 & 0.0 & 0.000 & 0.000 & 0.000 \\
\hline 110 & 0.000 & 0.000 & 0.000 & 0.000 & 0.000 & 0.000 & 0.154 & 95 & 0.000 & 0.000 & 0.000 & 0.0 & 0.000 & 1.000 & 0.000 \\
\hline$A A T-1^{\circ}$ & & & & & & & & 100 & 1.000 & 1.000 & 0.000 & 1.000 & 1.000 & 0.000 & 0.000 \\
\hline$(\mathrm{N})$ & (35) & (50) & $(48)$ & (33) & $(65)$ & $(48)$ & (45) & & 0.000 & 0.000 & 0.000 & 0.000 & 0.000 & 0.000 & 1.000 \\
\hline-50 & 0.000 & 0.000 & 1.000 & 1.000 & 0.000 & 0.000 & 0.000 & & & & & & & & \\
\hline-20 & 0.000 & 1.000 & 0.000 & 0.000 & 0.000 & 0.000 & 0.000 & I & & & & & $(40)$ & $(48)$ & \\
\hline-15 & 0.000 & 0.000 & 0.000 & 0.000 & 0.000 & 0.000 & 0.022 & & (30) & & & & $\begin{array}{c}(40) \\
0.000\end{array}$ & $\begin{array}{l}(48) \\
1.000\end{array}$ & \\
\hline 0 & 0.014 & 0.000 & 0.000 & 0.000 & 0.000 & 0.000 & 0.000 & & 0.000 & 0.0 & 0.000 & & $\begin{array}{l}0.000 \\
0.000\end{array}$ & 0.000 & \\
\hline 60 & 0.000 & 0.000 & 0.000 & 0.000 & 0.000 & 0.008 & 0.967 & & 0 & 1.0 & & & $\begin{array}{l}0.000 \\
1.000\end{array}$ & 0.000 & \\
\hline 70 & 0.000 & 0.000 & 0.000 & 0.000 & 1.000 & 0.000 & 0.000 & & 0 & .000 & 10 & 0 & & & \\
\hline 100 & 0.986 & 0.000 & 0.000 & 0.000 & 0.000 & 0.000 & 0.000 & $L A P$ & & & & & & & \\
\hline 125 & 0.000 & 0.000 & 0.000 & 0.000 & 0.000 & 0.000 & 0.011 & & $(30)$ & $(30)$ & (30) & (33) & $(40)$ & $(48)$ & (21) \\
\hline 130 & 0.000 & 0.000 & 0.000 & 0.000 & 0.000 & 0.992 & 0.000 & 100 & 1.000 & 1.000 & 1.000 & 1.000 & 0.000 & 1.000 & 0.000 \\
\hline$A A T-2$ & & & & & & & & 130 & 0.000 & 0.000 & 0.000 & 0.000 & 0.104 & 0.000 & 0.000 \\
\hline (N) & $(35)$ & $(50)$ & $(48)$ & (33) & $(65)$ & (48) & (44) & 140 & 0.000 & 0.000 & 0.000 & 0.000 & 0.896 & 0.000 & 0.000 \\
\hline 90 & 0.000 & 0.000 & 0.000 & 0.000 & 0.000 & 1.000 & 0.000 & 180 & 0.000 & 0.000 & 0.000 & 0.000 & 0.000 & 0.000 & 1.000 \\
\hline 100 & 1.000 & 1.000 & 0.000 & 0.000 & 0.000 & 0.000 & 1.000 & FUN & & & & & & & \\
\hline 105 & 0.000 & 0.000 & 0.000 & 0.000 & 1.000 & 0.000 & 0.000 & & (30) & (30) & $(30)$ & (33) & (42) & $(40)$ & $(38)$ \\
\hline 110 & 0.000 & 0.000 & 1.000 & 0.000 & 0.000 & 0.000 & 0.000 & 50 & 0.000 & 0.000 & 0.000 & 0.000 & 1.000 & 0.000 & 0.000 \\
\hline 115 & 0.000 & 0.000 & 0.000 & 1.000 & 0.000 & 0.000 & 0.000 & 70 & 0.000 & 0.000 & 0.000 & 1.000 & 0.000 & 0.000 & 0.000 \\
\hline$M D F$ & & & & & & & & 80 & 0.000 & 1.000 & 0.000 & 0.000 & 0.000 & 1.000 & 0.000 \\
\hline$(\mathrm{N})$ & (30) & $(30)$ & $(30)$ & (33) & $(45)$ & (45) & (21) & 85 & 0.000 & 0.000 & 0.000 & 0.000 & 0.000 & 0.000 & 0.092 \\
\hline 85 & 0.000 & 0.000 & 0.000 & 0.000 & 0.000 & 1.000 & 0.000 & 90 & 0.000 & 0.000 & 1.000 & 0.000 & 0.000 & 0.000 & 0.000 \\
\hline 87 & 0.000 & 0.000 & 0.000 & 0.000 & 1.000 & 0.000 & 0.000 & 95 & 0.000 & 0.000 & 0.000 & 0.000 & 0.000 & 0.000 & 0.184 \\
\hline 90 & 0.000 & 1.000 & 0.000 & 0.000 & 0.000 & 0.000 & 0.000 & 100 & 1.000 & 0.000 & 0.000 & 0.000 & 0.000 & 0.000 & 0.000 \\
\hline 95 & 0.000 & 0.000 & 1.000 & 0.000 & 0.000 & 0.000 & 0.000 & 105 & 0.000 & 0.000 & 0.000 & 0.000 & 0.000 & 0.000 & 0.724 \\
\hline
\end{tabular}


Table 3 (continued)

\begin{tabular}{|c|c|c|c|c|c|c|c|}
\hline $\begin{array}{l}\text { Locus, } \\
\text { Alleles }\end{array}$ & 1 & 2 & 3 & $\begin{array}{c}\text { Species } \\
4\end{array}$ & 5 & 6 & 7 \\
\hline \multicolumn{8}{|l|}{$H K^{*}$} \\
\hline 80 & 0.000 & 0.000 & 0.000 & 0.000 & 0.000 & 1.000 & 0.000 \\
\hline 82 & 0.000 & 0.000 & 0.000 & 0.017 & 0.000 & 0.000 & 0.000 \\
\hline 85 & 0.000 & 0.000 & 0.000 & 0.000 & 1.000 & 0.000 & 0.000 \\
\hline 90 & 0.000 & 1.000 & 1.000 & 0.983 & 0.000 & 0.000 & 0.000 \\
\hline 95 & 0.000 & 0.000 & 0.000 & 0.000 & 0.000 & 0.000 & 1.000 \\
\hline 100 & 1.000 & 0.000 & 0.000 & 0.000 & 0.000 & 0.000 & 0.000 \\
\hline \multicolumn{8}{|c|}{$D I A-1$} \\
\hline$(\mathrm{N})$ & (30) & $(30)$ & (30) & (33) & (45) & $(40)$ & (21) \\
\hline 95 & 0.000 & 0.000 & 0.000 & 0.000 & 0.000 & 0.000 & 1.000 \\
\hline 100 & 1.000 & 0.000 & 0.000 & 0.000 & 0.000 & 0.000 & 0.000 \\
\hline 105 & 0.000 & 1.000 & 1.000 & 1.000 & 1.000 & 1.000 & 0.000 \\
\hline \multicolumn{8}{|l|}{$D I A-2^{\circ}$} \\
\hline$(\mathrm{N})$ & $(30)$ & $(30)$ & (30) & (33) & (45) & $(40)$ & (21) \\
\hline 80 & 0.000 & 0.000 & 0.000 & 0.000 & 0.000 & 1.000 & 0.000 \\
\hline 90 & 0.000 & 0.000 & 0.000 & 0.000 & 0.000 & 0.000 & 1.000 \\
\hline 95 & 0.000 & 0.000 & 0.000 & 1.000 & 0.000 & 0.000 & 0.000 \\
\hline 100 & 1.000 & 0.000 & 0.000 & 0.000 & 1.000 & 0.000 & 0.000 \\
\hline 105 & 0.000 & 0.000 & 1.000 & 0.000 & 0.000 & 0.000 & 0.000 \\
\hline 110 & 0.000 & 1.000 & 0.000 & 0.000 & 0.000 & 0.000 & 0.000 \\
\hline \multicolumn{8}{|l|}{ DIA-3. } \\
\hline$(N)$ & $(30)$ & $(30)$ & (30) & (33) & (45) & $(40)$ & (21) \\
\hline 90 & 0.000 & 0.000 & 0.000 & 0.000 & 1.000 & 0.000 & 0.000 \\
\hline 95 & 0.000 & 0.000 & 0.000 & 1.000 & 0.000 & 0.000 & 1.000 \\
\hline 100 & 1.000 & 1.000 & 1.000 & 0.000 & 0.000 & 1.000 & 0.000 \\
\hline \multicolumn{8}{|l|}{$A C P^{\circ}$} \\
\hline 80 & 0.000 & 0.000 & 0.000 & 0.000 & 0.230 & 0.000 & 0.000 \\
\hline 100 & 1.000 & 0.000 & 0.000 & 0.000 & 0.690 & 1.000 & 0.000 \\
\hline 105 & 0.000 & 0.000 & 1.000 & 1.000 & 0.000 & 0.000 & 0.000 \\
\hline 110 & 0.000 & 0.000 & 0.000 & 0.000 & 0.070 & 0.000 & 1.000 \\
\hline 120 & 0.000 & 1.000 & 0.000 & 0.000 & 0.010 & 0.000 & 0.000 \\
\hline \multicolumn{8}{|l|}{ PEP-1 } \\
\hline$(\mathrm{N})$ & $(30)$ & $(30)$ & $(30)$ & (33) & (39) & (45) & (37) \\
\hline 50 & 0.000 & 0.000 & 0.000 & 0.000 & 0.000 & 1.000 & 0.000 \\
\hline 90 & 0.000 & 0.000 & 0.000 & 0.076 & 1.000 & 0.000 & 0.000 \\
\hline 95 & 0.000 & 1.000 & 0.000 & 0.000 & 0.000 & 0.000 & 0.000 \\
\hline 100 & 1.000 & 0.000 & 1.000 & 0.924 & 0.000 & 0.000 & 0.000 \\
\hline 110 & 0.000 & 0.000 & 0.000 & 0.000 & 0.000 & 0.000 & 1.000 \\
\hline \multicolumn{8}{|c|}{ PEP-2. } \\
\hline$(\mathrm{N})$ & $(30)$ & $(30)$ & $(30)$ & (33) & (39) & $(45)$ & $(37)$ \\
\hline 75 & 0.000 & 0.000 & 0.000 & 0.000 & 1.000 & 0.000 & 0.000 \\
\hline 80 & 0.000 & 0.000 & 0.000 & 0.000 & 0.000 & 1.000 & 0.000 \\
\hline 85 & 0.000 & 0.000 & 0.000 & 0.000 & 0.000 & 0.000 & 0.932 \\
\hline 95 & 0.000 & 0.000 & 1.000 & 1.000 & 0.000 & 0.000 & 0.000 \\
\hline 100 & 1.000 & 1.000 & 0.000 & 0.000 & 0.000 & 0.000 & 0.000 \\
\hline 105 & 0.000 & 0.000 & 0.000 & 0.000 & 0.000 & 0.000 & 0.068 \\
\hline
\end{tabular}

All the species were successfully analysed for 13 enzymes coded by a total of 21 loci. Allele frequencies for all species studied are shown in Table 3. Only 4 loci (GPI*, PGM ${ }^{*}, \mathrm{MPI}^{*}$, and AAT-1*) were consistently polymorphic ( 0.99 criterion) in several or most of the species. FUM ${ }^{*}$ and PEP-2* were also polymorphic in Gammarus crinicornis, $H K^{*}$ and $P E P-1^{*}$ in Orchestia mediterranea, and $L A P-2^{\circ}$ and $A C P^{*}$ in Arcitalitrus
Table 4. Mean heterozygosities (H) (SE in parentheses), the mean number of alleles per locus, and percentage of polymorphic loci (P) 10.99 criterion) in the 7 amphipod species studied

\begin{tabular}{|c|c|c|c|c|}
\hline Species & Observed & Expected & $\begin{array}{l}\text { Mean no. } \\
\text { alleles }\end{array}$ & $\mathrm{P}$ \\
\hline $\begin{array}{l}\text { Talitrus } \\
\text { saltator }\end{array}$ & $\begin{array}{c}0.020 \\
(0.014)\end{array}$ & $\begin{array}{c}0.024 \\
(0.016)\end{array}$ & 1.3 & 14.3 \\
\hline $\begin{array}{l}\text { Talorchestia } \\
\text { deshayesii }\end{array}$ & $\begin{array}{c}0.056 \\
(0.038)\end{array}$ & $\begin{array}{c}0.047 \\
(0.032)\end{array}$ & 1.2 & 14.3 \\
\hline $\begin{array}{l}\text { Orchestia } \\
\text { gammarellus }\end{array}$ & $\begin{array}{c}0.015 \\
(0.013)\end{array}$ & $\begin{array}{c}0.016 \\
(0.014)\end{array}$ & 1.2 & 14.3 \\
\hline $\begin{array}{l}\text { Orchestia } \\
\text { mediterranea }\end{array}$ & $\begin{array}{c}0.030 \\
(0.016\}\end{array}$ & $\begin{array}{c}0.031 \\
(0.015)\end{array}$ & 1.3 & 23.8 \\
\hline $\begin{array}{l}\text { Arcitalitrus } \\
\text { dorrieni }\end{array}$ & $\begin{array}{c}0.025 \\
(0.017)\end{array}$ & $\begin{array}{c}0.033 \\
(0.024)\end{array}$ & 1.2 & 14.3 \\
\hline $\begin{array}{l}\text { Hyale } \\
\text { nilssoni }\end{array}$ & $\begin{array}{c}0.014 \\
(0.011)\end{array}$ & $\begin{array}{c}0.013 \\
(0.010)\end{array}$ & 1.1 & 14.3 \\
\hline $\begin{array}{l}\text { Gammarus } \\
\text { crinicornis }\end{array}$ & $\begin{array}{c}0.059 \\
(0.027)\end{array}$ & $\begin{array}{c}0.070 \\
(0.031)\end{array}$ & 1.5 & 28.6 \\
\hline
\end{tabular}

Table 5. Estimates of Nei's (1978) genetic identity (above the diagonal) and genetic distance (below the diagonal) between the 7 amphipod species. (1) Talitrus saltator, (2) Talorchestia deshayesii, (3) Orchestia gammarellus, (4) Orchestia mediterranea, (5) Arcitalitrus dorrieni, (6) Hyale nilssoni, (7) Gammarus crinicornis.

\begin{tabular}{|cccccccc|}
\hline Species & 1 & 2 & 3 & 4 & 5 & 6 & 7 \\
\hline 1 & $\ldots \ldots$ & 0.347 & 0.235 & 0.325 & 0.270 & 0.237 & 0.141 \\
2 & 1.058 & $\cdots \cdots$ & 0.352 & 0.359 & 0.249 & 0.246 & 0.152 \\
3 & 1.448 & 1.045 & $\ldots \ldots$ & 0.560 & 0.145 & 0.195 & 0.100 \\
4 & 1.125 & 1.025 & 0.580 & $\cdots \cdots$ & 0.192 & 0.146 & 0.150 \\
5 & 1.309 & 1.392 & 1.931 & 1.652 & $\ldots \ldots$ & 0.131 & 0.055 \\
6 & 1.439 & 1.402 & 1.637 & 1.922 & 2.032 & $\cdots \cdots$ & 0.100 \\
7 & 1.960 & 1.885 & 2.307 & 1.894 & 2.900 & 2.305 & $\cdots \cdots$ \\
\hline
\end{tabular}

dorrieni. The remaining loci were monomorphic within species, but were in many cases fixed for alternative alleles in different species.

Table 4 summarises the results for mean number of alleles per locus, percentage of loci polymorphic, and mean heterozygosity for each species studied. The observed heterozygosity ranged from 0.014 in Hyale nilssoni to 0.059 in Gammarus crinicornis. The matrix of genetic similarity and distance coefficients (Nei 1978 ) is shown in Table 5. The values for genetic identity ranged from a very low value of 0.055 between $G$. crinicornis and Arcitalitrus dorrieni to 0.560 between the congeneric species Orchestia gammarellus and $O$. mediterranea.

Both dendrograms (Fig. 2a, b) suggest that Talorchestia deshayesii, a sandhopper, is more closely related to the beachfleas (Orchestia gammarellus and $O$. mediterranea) than to Talitrus saltator, the other sand- 

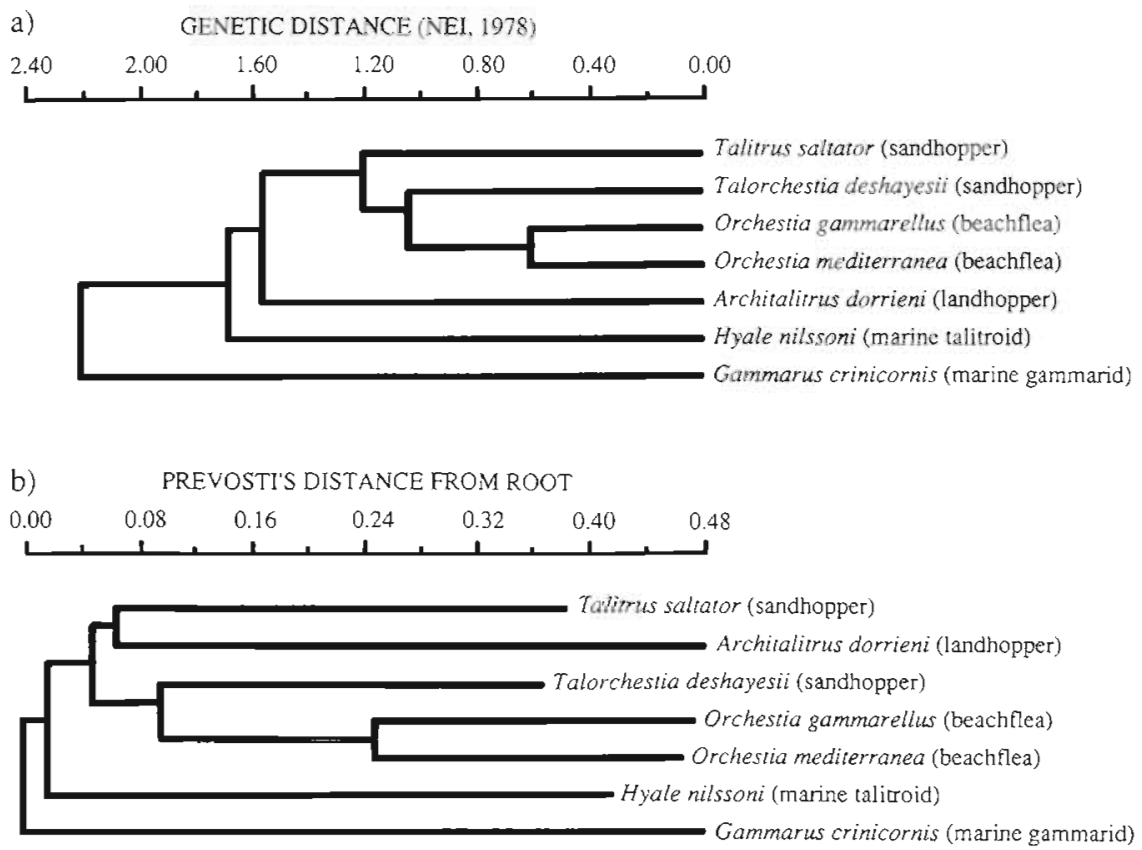

\begin{abstract}
Fig. 2. Dendrograms to indicate interrelationships of the 7 amphipod species studied (a) derived by the UPGMA method using Nei's genetic distance and (b) by the Wagner procedure using Prevosti's distance. Goodness of fit statistics: (a) $f=4.312$, $\% S D=14.08, F=12.59, C C=0.870$ (b) $f=0.404, \% S D=4.74, F=2.46$, $C C=0.969$. For further information and references to statistical methodology see 'Materials and methods'
\end{abstract}

hopper species. The main difference between the dendrogram obtained by the Wagner distance procedure (Fig. 2b) and that obtained by the UPGMA analysis is that Arcitalitrus dorrieni was grouped with $T$. saltator in the Wagner dendrogram, whereas in the UPGMA dendrogram (Fig. 2a) A. dorrieni is the most divergent of the talitrid species studied. The goodness of fit statistics (see Fig. 2a, b) indicate that the Wagner distance procedure produced the better tree.

\section{DISCUSSION}

\section{Levels of genetic variation}

The heterozygosity values for the talitrid species studied (Table 4) are low when compared with published data for several other amphipod species (e.g. Battaglia et al. 1978, Dickson et al. 1979, Gooch \& Hetrick 1979, Siegismund et al. 1985, Kane et al. 1992, Patarnello et al. 1992) and when compared with estimates for other crustaceans (Hedgecock et al. 1982). Bulnheim \& Scholl (1986) and De Mathaeis et al. (1994) have also reported low levels of genetic variability for Talitrus saltator and Talorchestia deshayesii. Thus it appears that talitrids may have generally rather low levels of heterozygosity. Levels of heterozygosity vary greatly over the wide range of organisms surveyed (Nevo 1978, Nevo et al. 1984), but the implications of this variation are far from clear (review by e.g. Zouros \& Foltz 1987). Ward et al. (1992) found that for invertebrates about $34 \%$ of variance in average protein het- erozygosity was attributable to taxon effects and $41 \%$ to the effects of protein structure and function.

The landhopper Arcitalitrus dorrieni is native to south-east Australia and is thought to have been introduced to the British Isles via imported plants (Richardson 1980). The various isolated colonies of $A$. dorrieni in Britain probably arose from relatively few introduced individuals; hence, a population bottleneck (founder effect) and consequently reduced levels of allozyme heterozygosity are to be expected. Although the heterozygosities of PGM* and GPI" are relatively low, the mean level of heterozygosity found for $A$. dorrieni was similar to that of the native species studied. (A. dorrieni was polymorphic for the otherwise monomorphic loci $L A P-1^{*}$ and $A C P$ *.) However, bottlenecks are believed to have a larger effect on allelic diversity than on heterozygosity, and if population size increases rapidly following a single bottleneck of short duration, the loss in mean heterozygosity may be small (Nei et al. 1975, Leberg 1992). In order to establish the significance of the pattern of genetic variation observed in $A$. dorrieni, it would be necessary to compare the results with genetic variation of putative ancestral Australian populations

\section{Genetic relationship between species}

The distribution of genetic identities between the species studied corresponds with the general relationship expected between taxonomic divergence and genetic identity (Thorpe 1982). The genetic identity for 
the 2 Orchestia species (Table 5) is within the range proposed for congeneric species (0.35 to 0.85$)$, and the values of genetic identity among the other different genera are around 0.35 or below, also as would be predicted.

However, in a review of genetic data from amphipods, Stewart (1993) suggested that, when identity values fell between 0.45 and 0.85 , it may be difficult to make decisions regarding specific status of populations, and additional factors such as evidence of a lack of gene flow and concordant morphological variation should be considered. The poor distinction found by Stewart is possibly due to a taxonomic effect, since most of the data came from the single genus Gammarus or other Gammaridae. It appears from the data summarised by Stewart (1993) that some Gammarus species are only about as closely related as talitrid genera.

Our genetic results do not indicate a clear relationship between Bousfield's systematic-ecological groups of talitrids and their genetic divergence, since both the UPGMA and the Wagner procedure dendrograms (Fig. 2a, b) suggest that the genera Orchestia (beachflea) and Talorchestia (sandhopper) are more closely related to each other than to Talitrus (sandhopper). This differs from the relationship in the phenogram of Bousfield $(1982,1984)$. However, the relative isolation of Talitrus saltator within the family has also been commented on by Hurley (1975) and Moore et al. (1993), whilst Bousfield (1984) stressed that his groups were not necessarily monophyletic.

As outlined above, the genetic relationships among all the species shown by a UPGMA dendrogram using Nei's genetic distance (Fig 2a) suggest that the landhopper Arcitalitrus dorrieni is the most divergent of the talitrid species studied. However, results from the Wagner procedure, which has stronger statistical support than the UPGMA tree (see Fig. 2a, b), show a different branching pattern for $A$. dorrieni. In the Wagner dendrogram $A$. dorrieni was clustered, albeit loosely, with Talitrus saltator, suggesting that both species share a direct common ancestor. It should be noted, however, that none of our results indicates a particularly close relationship between $A$. dorrieni and $T$. saltator, the type-species of Talitrus, despite the referral of $A$. dorrieni and other simplidactylate landhoppers to Talitrus (sensu lato) by some authors (e.g. Hurley 1975, Richardson 1980). Bousfield (1984) suggested that simplidactylate landhoppers evolved from an ancestral palustral species, although Morritt (1988) and Moore et al. (1993) hypothesised an evolutionary pathway from the hyalids to the simplidactylate landhoppers without a palustral species as intermediary.

Our results also indicate that Hyale nilssoni is more closely related to talitrids than is Gammarus crinicor- nis. H. nilssoni was formerly placed in the family Talitridae, but, largely on ecological criteria, has been referred to the family Hyalidae within the superfamily Talitroidea (Bulycheva 1957). This is compatible with the genetic data presented here. The data also provide limited support for the hypothesis that the talitrids may have originated from aquatic ancestors allied to primitive hyalids (e.g. Bousfield 1984).

The degree of genetic divergence reported here between the ecomorphological groups of the Talitridae is unexpectedly small in the light of Bousfield's (1984) evolutionary scenario (see also Hurley 1975), which proposed an ancient origin of simplidactylate landhoppers, with a divergence from other groups around 100 million years before present, during the Cretaceous period. This long divergence is not easily reconciled with the values of Nei's (1978) genetic identity, which indicate far more recent divergence. Calibrations of molecular clocks are controversial and at best inaccurate, but our data would generally be considered to indicate that the major divergence of the Talitridae has probably occurred over about the last 10 million years (see Thorpe 1982, 1989, Nei 1987), and thus almost certainly commenced within Neogene (late Cenozoic) times (ca 2 to 24 million yr before present).

Overall, therefore, our data, when compared with previous hypotheses, indicate some small but significant differences in patterns of divergence of the Talitridae, but also show a degree of general agreement, although they are not compatible with the very long timescale proposed in the major study by Bousfield (1984). To clarify further the phylogeny of the talitrids it would be desirable to include some palustral species and cuspidactylate landhoppers in the analysis and also to increase the number of species in each ecological group.

Acknowledgements. M.B.C. received financial support from the Brasilian Government, through a PhD grant from CAPES, and J.D.D.B. was in receipt of an Advanced Research Fellowship from the UK Natural Environment Research Council. These awards are gratefully acknowledged.

\section{LITERATURE CITED}

Barnard JL (1974) Evolutionary patterns in gammaridean Amphipoda. Crustaceana 27:137-146

Battaglia B, Bisol PM, Fava G (1978) Genetic variability in relation to the environment in some marine invertebrates In: Battaglia B, Beardmore JA (eds) Marine organisms, genetics, ecology and evolution. Plenum Press, London, p $53-70$

Bousfield EL. (1982) The amphipod superfamily Talitroidea in the northeasthern Pacific Region. I. Family Talitridae: systematics and distributional ecology. Natl Mus Nat Sci (Ott) Publ Biol Oceanogr 11:1-75

Bousfield EL (1984) Recent advances in the systematics and biogeography of landhoppers (Amphipoda: Talitridae) of 
the Indo-Pacific Region. In: Radovsky FJ, Raven PH, Sohmer SH (eds) Biogeography of the tropical Pacific Bernice P Bishop Mus Spec Publ 72:171-210

Bulnheim HP, Scholl A (1986) Genetic differentiation between populations of Talitrus saltator and Talorchestia deshayesii (Crustacea: Amphipoda) from coastal areas of the north-western European continent. Mar Biol 92: $525-536$

Bulycheva Al (1957) Morskie bloxi moreì SSSR i sopredel'nyx vod (Amphipoda, Talitroidea). Opred Po Faune SSSR, Akad Nauk SSSR 65:1-186

Conceição MB (1995) Genetic investigation of talitrid amphipods. PhD thesis, University of Liverpool

De Matthaeis E, Cobolli M, Mattoccia M, Saccoccio P, Scapini $F$ (1994) Genetic divergence between natural populations of Mediterranaean sandhoppers (Crustacea, Amphipoda). In: Beaumont AR (ed) Genetics and evolution of aquatic organisms. Chapman \& Hall, London, p 15-29

Dickson GW, Patton JC, Holsinger JR, Avise JC (1979) Genetic variation in cave-dwelling and deep-sea organisms, with emphasis on Crangonyx antennatus (Crustacea: Amphipoda) in Virginia. Brimleyana 2:119-130

Farris JS (1972) Estimating phylogenetic trees from distance matrices. Am Nat 106:645-668

Fitch WM, Margoliash E (1967) Construction of phylogenetic trees. Science 155:279-284

Gooch JL, Hetrick SW (1979) The relation of genetic structure to environmental structure: Gammarus minus in a Karst area. Evolution 33:192-206

Griffiths CL, Stenton-Dozey J (1981) The fauna and rate of degradation of stranded kelp. Estuar Coast Shelf Sci 12 : 645-653

Harris H, Hopkinson DA (1976) Handbook of enzyme electrophoresis in human genetics. North-Holland, Amsterdam

Hedgecock D, Tracey ML, Nelson K (1982) Genetics. In: Abele LG (ed) Biology of the Crustacea, Vol 2. Academic Press, New York, p 283-403

Hurley DE (1959) Notes on the ecology and environmental adaptations of the terrestrial Amphipoda. Pac Sci 13: $107-129$

Hurley DE (1975) A possible subdivision of the terrestrial genus Talitrus (Crustacea Amphipoda: family Talitridae). NZOI Rec 2:157-170

Kane TC, Culver DC, Jones RI (1992) Genetic structure of morphologically differentiated populations of the amphipod Gammarus minus. Evolution 46:272-278

Kim CB, Kim W (1993) Phylogenetic relationships among gammaridean families and amphipod suborders. J Nat Hist 27:933-946

Koop K, Newell RC, Lucas MI (1982a) Biodegradation and carbon flow based on kelp (Ecklonia maxima) debris in a sandy beach microcosm. Mar Ecol Prog Ser 7:315-326

Koop K, Newell RC, Lucas MI (1982b) Microbial regeneration of nutrients from the decomposition of macrophyte debris on the shore. Mar Ecol Prog Ser 9:91-96

Leberg PL (1992) Effects of population bottlenecks on genetic diversity as measured by allozyme electrophoresis. Evolution $46: 477-494$

Levinton JS, Lopes GR, Lassen HH, Rahn. U (1977) Feedback and structure in deposit feeding marine benthic communities. In: Keegan BF, O'Ceidigh P, Boaden PS (eds) Biology of benthic organisms. Pergamon Press, Oxford, p 409-416

Lindeman D (1991) Phylogeny and zoogeography of the new world terrestrial amphipods (landhoppers) (Crustacea: Amphipoda: Talitridae). Can J Zool 69:1104-1116

McDonald JH (1985) Size-related and geographical variation at two enzyme loci in Megalorchestia californiana (Amphipoda: Talitridac). Heredity 54:359-366

McDonald JH (1987) Repeated geographical variation at three enzyme loci in the amphipod Platorchestia platensis. Evolution 41:438-441

McDonald JH (1991) Selection component analysis of the MPI locus in the amphipod Platorchestia platensis. Heredity 62:243-249

Moore PG, Francis CH (1985) Some observations on food and feeding of the supralittoral beach-hopper Orchestia gammarellus (Pallas) (Crustacea: Amphipoda). Ophelia 24 183-197

Moore PG, Morrit D, Stevenson TDI (1993) Morphology of the antennary gland exit duct in ecological and phylogenetic series of talitroid Amphipoda (Crustacea). J Zool (Lond) 231:187-201

Morrit D (1988) Osmoregulation in littoral and terrestrial talitroidean amphipods (Crustacea) from Britain. J Exp Mar Biol Ecol 123:77-94

Murphy RW, Sites JW, Buth DG, Haufler CH (1990) Proteins I. isozyme electrophoresis. In: Hillis DM, Moritz C (eds) Molecular systematics. Sinauer, Sunderland, MA, p 45-126

Nei M (1978) Estımation of average heterozygosity and genetic distance from a small number of individuals. Genetics 89:583-590

Nei M (1987) Molecular evolutionary genetics. Columbia University Press. New York.

Nei M, Maruyama T, Chakraborty R (1975) The bottleneck effect and genetic variability in populations. Evolution 29 $1-10$

Nevo $E$ (1978) Genetic variation in natural populations: patterns and theory. Theor Pop Biol 13:121-177

Nevo E, Beiles A, Ben-Shlomo R (1984) The evolutionary significance of genetic diversity: ecological, demographic and life history correlates. In: Mani GS (ed) Evolutionary dynamics of genetic diversity. Springer-Verlag, Berlin, p 13-213

Patarnello T, Battaglia B, Bisol PM (1992) Genetic differentiation among geographic populations of two species of the genus Gammarus: $G$. insensibilis and $G$. aequicauda (Crustacea: Amphipoda). Vie Milieu 42:263-268

Poulik MD (1957) Starch gel electrophoresis in a discontinuous system of buffers. Nature 180:1477-1479

Prager EM, Wilson AC (1976) Congruency of phylogenies derived from different proteins. J Mol Evol 9:45-57

Richardson AMM (1980) Notes on the ocurrence of Talitrus dorrieni Hunt (Crustacea: Amphipoda: Talitridae) in south-west England. J Nat Hist 14:751-757

Robertson AI, Lucas JS (1983) Food choice, feeding rates, and the turnover of macrophyte biomass by a surf-zone inhabiting amphipod. J Exp Mar Biol Ecol 72:99-124

Shaklee JB, Keenam CP (1986) A practical laboratory guide to the techniques and methodology of electrophoresis and its application to fish fillet identification, Report 177. CSIRO, Melbourne

Siciliano MJ, Shaw CR (1976) Separation and visualization of enzymes on gels. In: Smith I (ed) Chromatographic and electrophoretic techniques, Vol 2. Halsted Press, London, p 185-209

Siegismund HR, Simonsen V, Kolding S (1985) Genetic studies of Gammarus. I. Genetic differentiation of local populations. Hereditas 102:1-13

Sneath PHA. Sokal RR (1973) Numerical taxonomy. W H Freeman, San Francisco

Spicer JI, Moore PG, Taylor AC (1987) The physiological ecology of land invasion by the Talitridae (Crustacea: Amphipoda). Proc R Soc Lond (Ser B) 232:95-124 
Stewart BA (1993) The use of protein electrophoresis for determining species boundaries in amphipods. Crustaceana 65:265-277

Swofford DL (1981) On the utility of the Distance Wagner Procedure. In: Funk VA, Brooks DR (eds) Advances in cladistics: proceedings of the first meeting of the Willi Hennig Society. New York Botanical Garden, New York, p 25-43

Swofford DL, Selander RB (1981) BIOSYS-1: a FORTRAN program for the comprehensive analysis of electrophoretic data in population genetics and systematics. J Hered 72 : $281-283$

Thorpe JP (1982) The molecular clock hypothesis: biochemlcal evolution, genetic differentiation and systematics. Annu Rev Ecol Syst 13:139-168

Thorpe JP (1989) Possible effects of interprotein variation in mean rate of amino acid substitution on the relationship of genetic distance with time since evolutionary divergence. Biol J Linn Soc 37:335-344

Editorial responsibility: Otto Kinne (Editor),

Oldendorf/Luhe, Germany
Thorpe JP, Solé-Cava AM (1994) The use of allozyme electrophoresis in invertebrate systematics. Zool Scripta 23:3-18

Venables BJ (1981) Aspects of the population biology of a Venezuelan beach amphipod, Talorchestia margaritae (Talitridae) including estimates of biomass and daily production, and respiration rates. Crustaceana 41:271-285

Ward RD, Skibinski DOF, Woodwark M (1992) Protein heterozygosity, protein structure, and taxonomic differentiation. Evol Biol 26:73-159

Wildish DJ (1988) Ecology and natural history of aquatic Talitroidea. Can J Zool 66:2340-2359

Wright S (1978) Evolution and the genetics of populations Vol 4, Variability within and among natural populations. University of Chicago Press, Chicago

Zouros E, Foltz DW (1987) The use of allelic isozyme variation for the study of heterosis. In: Rattazzi MC, Scandalios JC, Whitt GS (eds) Isozymes. Current topics in biological and medical research, Vol 13. Alan R Liss, New York, p 2-59

Submitted: February 12, 1997; Accepted: November 10, 1997 Proofs received from author(s): April 24, 1998 